\title{
Evaluación del estado metabólico, el peso vivo y la condición corporal durante la gestación en vacas de carne en la Altillanura colombiana
}

\author{
Evaluation of the metabolic status, live weight and body condition \\ during pregnancy in beef in the Colombian Altillanura cows
}

\section{Avaliação do estado metabólico, peso vivo e condição corporal durante a gestação em vacas de corte no Altillanura colombiana}

\author{
Gerardo López-García ${ }^{1}$, Agustín Góngora-Orjuela ${ }^{2}$, Félix Díaz-González ${ }^{3}$, \\ Adriana Rojas-Rodríguez ${ }^{4}$ \\ 1 MVZ, Esp, MSc, Est. Doctorado en Cienc Vet, Becario Escuela Graduados Facultad de Ciencias Veterinarias. Universidad \\ Austral de Chile, Valdivia. \\ 2 MV, MSc, PhD, Universidad de los Llanos, grupo de investigación GIRGA, Facultad de Ciencias Agropecuarias y Recursos \\ Naturales \\ 3 MV, MSc, PhD, Universidade Federal do Rio Grande do Sul, Brasill \\ 4 MV, Esp, MSc, Universidad Cooperativa de Colombia \\ Email: gerardo.lopez@alumnos.uach.cl
}

Recibido: 29 de noviembre de 2014

Aceptado: 26 de marzo de 2019

\begin{abstract}
Resumen
El objetivo de este estudio, fue conocer los valores promedios de diferentes metabolitos, la variación del peso vivo (PV) y la condición corporal (CC) en vacas Cebú (C) y Simmental x Cebú (SC) bajo pastoreo extensivo en una finca de la Altillanura colombiana. Se utilizaron 19 vacas multíparas y durante cada trimestre de la gestación, se evaluaron los niveles de Glucosa (Glu), Colesterol (Col), Proteína totales (Ptt), Albúmina (Alb), Globulinas (Glo), Hemoglobina $(\mathrm{Hb})$, Hematocrito (Hto), Nitrógeno en sangre (BUN), Cobre (Cu), Zinc (Zn), Calcio (Ca), Magnesio (Mg), Fósforo (P), y Aspartato aminotransferasa (AST). Los niveles de Glu, Hb, Hto, BUN, Ca, presentaron variaciones significativas durante los trimestre evaluados $(\mathrm{P}<0,05)$, con deficiencias de $\mathrm{Cu}$ y $\mathrm{Zn}$. El PV y la CC presentaron una relación lineal en función del avance de la gestación. Se reconoce la importancia de los perfiles metabólicos (PM) como una herramienta para identificar los posibles imbalances nutricionales y la manera de corregir las deficiencias, con el propósito de hacer mas eficiente el sistema productivo.
\end{abstract}

Palabras clave: bioquímica sanguínea; condición corporal; minerales; producción bovina; vacas gestantes 


\begin{abstract}
The objective of this study was to know the average values of different metabolites, the variation of live weight (LW) and body condition (CC) in Cebu (C) and Simmental x Cebu (SC) cows under extensive grazing in a farm of the Colombian Altillanura. We used 19 multiparous cows and during each trimester of pregnancy, we evaluated the levels of Glucose (Glu), Cholesterol (Col), Total Protein (Ptt), Albumin (Alb), Globulins (Glo), Hemoglobin (Hb), Hematocrit (Hto), Nitrogen in blood (BUN), Copper (Cu), Zinc (Zn), Calcium (Ca), Magnesium (Mg), Phosphorus (P), and Aspartate aminotransferase (AST). The levels of Glu, Hb, Hto, BUN, Ca presented significant variations during the quarter evaluated $(\mathrm{P}<0.05)$, with deficiencies of $\mathrm{Cu}$ and $\mathrm{Zn}$. The PV and CC presented a linear relationship based on the progress of pregnancy. The importance of metabolic profiles (PM) is recognized as a tool to identify possible nutritional imbalances and how to correct deficiencies, in order to make the productive system more efficient.
\end{abstract}

Key words: blood chemistry; body condition; minerals; cattle production; pregnant cows

\begin{abstract}
Resumo
O objetivo deste estudo foi conhecer os valores médios dos diferentes metabólitos, a variação do peso vivo (PV) e a condição corporal (CC) em vacas Cebu (C) e Simmental x Cebu (SC) sob pastejo intenso numa fazenda da Altillanura colombiana. Foram utilizadas 19 vacas multíparas e, durante cada trimestre da gestação, foram avaliados os níveis de glicose (Glu), colesterol (Col), proteína total (Ptt), albumina (Alb), globulinas (Glo), hemoglobina (Hb) e hematócrito (Hto), nitrogênio sanguíneo $(\mathrm{BUN})$, cobre $(\mathrm{Cu})$, zinco $(\mathrm{Zn})$, cálcio $(\mathrm{Ca})$, magnésio $(\mathrm{Mg})$, fósforo $(\mathrm{P})$ e aspartato aminotransferase (AST). Os níveis de Glu, Hb, Hto, BUN, Ca apresentaram variações significativas durante os trimestres avaliados (P <0,05), com deficiências de Cu e Zn. O PV e o CC apresentaram uma relação linear com o progresso da gravidez. A importância dos perfis metabólicos (MP) é reconhecida como uma ferramenta para identificar possíveis desequilíbrios nutricionais e como corrigir deficiências, a fim de tornar o sistema de produção mais eficiente.
\end{abstract}

Palavras-chave: bioquímica sanguínea; condição corporal; minerais; produção bovina; vacas grávidas

\section{Introducción}

La Orinoquia es una vasta región del territorio colombiano (26 millones de hectáreas) que tiene una importancia estratégica para el desarrollo del país; más del $53 \%$ de este territorio corresponde a la Altillanura bien drenada (Mejía, 1984), allí se ubica una población de 664.604 animales especialmente de cría (Compes, 2014) que produce un alto porcentaje de machos para la ceba que surten los grandes centros de consumo del país.

El sistema de cría se maneja de forma extensiva y se ve afectado entre otros factores por la estacionalidad de las lluvias, la pobre fertilidad de los suelos, el bajo contenido de materia orgánica, los altos niveles de aluminio intercambiable, lo que lleva a una escasa disponibilidad de nutrientes para los forrajes, todo lo cual, se traduce en bajos niveles productivos (Rivas et al., 2004). Después de analizar diferentes especies forrajeras en la Altillanura, se encontraron deficiencias, especialmente de $\mathrm{Cu}$ y $\mathrm{Zn}$; estas mismas deficiencias se identificaron en los tejidos de los animales (Laredo y Gómez 1980; Gómez y Laredo 1983).

Durante la gestación en el bovino ocurren importantes cambios metabólicos, hormonales y reproductivos (Chilliard et al., 1998; Kindahl et al., 2002) que se ven influenciados por la nutrición, pero solo se reflejan al momento del parto o durante el posparto con la apa- rición de las enfermedades metabólicas o asociadas a la producción (Barth et al., 2011; Smith et al., 2017).

Los perfiles metabólicos (PM) se utilizaron por primera vez en ganado de leche en Inglaterra (Payne et al., 1970), desde entonces, se han constituido en una herramienta complementaria en el diagnóstico de las enfermedades asociadas a la producción (Quintella et al., 2011). El fundamento de esta técnica se basa en el análisis de ciertos componentes de la sangre como indicadores de imbalances nutricionales, lo que permite mejorar la alimentación, detectar problemas subclínicos y prevenir las enfermedades especialmente durante el periodo de transición (Butler y Smith, 1989). De forma paralela, la estimación de la condición corporal (CC) ha sido recomendada para evaluar el manejo de la nutrición (Rae et al., 1996) ya que permite medir la cantidad de energía metabólica almacenada en forma de grasa subcutánea y en el músculo (Houghton et al., 1990).

La variación en los cambios de la CC pueden reflejar el estado nutricional; en consecuencia una baja CC puede tener un valor predictivo de los trastornos metabólicos, fallas en la reproducción y la producción de leche (Edmonson et al., 1989). Por otro lado, la restricción energética durante la gestación disminuye la CC al parto y reduce el peso de los terneros al nacimiento (Freetly et al., 2000) por consiguiente el estado nutri- 
cional de la madre, es el principal factor en el desarrollo y función del sistema de órganos fetales (Caton y Hess, 2010). Igualmente, la baja ingestión de energía y proteína durante la gestación tardía, afecta el desarrollo de la placenta, que conduce al nacimiento de terneros débiles y un aumento en la retención de la placenta (Zhang et al., 2002).

El objetivo de este estudio fue conocer los valores de determinados metabolitos sanguíneos, la condición corporal y el peso vivo de vacas de cría, durante cada uno de los trimestres de gestación en una finca de nivel tecnológico medio de la Altillanura Colombiana.

\section{Materiales y métodos}

\section{Ubicación y Manejo del Sistema productivo}

El estudio se llevó a cabo en una finca ubicada en el municipio de Puerto López, Meta, Colombia ( $4^{\circ} 6^{\prime} 7^{\prime \prime}$ latitud N y $72^{\circ} 54^{\prime} 24^{\prime \prime}$ longitud Oeste), entre los meses de Enero-Diciembre de 2013. Se utilizaron 19 vacas multíparas de un grupo de 159, entre 3 a 4 partos de la raza Cebú (C) y cruces absorbentes de Cebú con Simmental $(\mathrm{CxS})$ con edades entre los 5 y 6 años. Los animales seleccionados fueron sometidos a un programa de inseminación a tiempo fijo (IATF) siguiendo el protocolo sugerido por Cutaia et al., (2003) y confirmada la preñez por ultrasonido al día 50 posinseminación (SSD-500 Aloka Co, Tokyo, Japan).

Los animales durante el estudio se mantuvieron en pastoreo continuo en praderas de Brachiaria humidicola cv. dyctioneura (pasto llanero), Brachiaria brizantha cv. marandú y leguminosas nativas perennes como Desmodium spp y Centrosema spp., sal mineralizada al $8 \%$ de fósforo y agua permanente.

\section{Toma de muestras, análisis de metabolitos, peso vivo y condición corporal}

De cada vaca se tomaron 2 muestras de $5.0 \mathrm{ml}$ por punción coccígea con y sin anticoagulante en tubos vacutainer estériles, transcurrido 30 minutos de su obtención, las muestras con anticoagulante fueron centrifugadas a $3.000 \mathrm{rpm}$ durante 10 minutos y el suero obtenido conservado a $-70^{\circ} \mathrm{C}$. El muestreo se realizó a las 7:00 horas para cada uno de los trimestres de gestación T1: gestación temprana (segunda semana del mes de mayo), T2: gestación media (segunda semana de Septiembre) y T3: gestación tardía (segunda semana de Diciembre). La Glu, Col, Alb, Ptt, Hb, Ca, P, Cu y $\mathrm{Mg}$ se determinaron por el método colorimétrico de punto final, mientras la urea, Zn y la AST mediante mé- todo cinético - enzimático, las Glo por la diferencia entre Ptt y Alb, Hto por tubo capilar. Los reactivos usados fueron Spinreact (Spinreact, Girona, España) y la lectura de las pruebas, se realizó en un espectrofotómetro semiautomatico (Mindray Ba-88 A, Shenzhen, China). Simultáneamente al muestreo sanguíneo se realizó el pesaje de las vacas en una báscula con capacidad de $1.000 \mathrm{~kg}$ (Prometalico) y evaluada la CC en escala de 1-9 en 1: emanciada y 9:obesa (Morrison et al., 1999)

\section{Análisis estadístico}

Los resultados fueron analizados mediante estadística descriptiva para cada una de las variables en estudio, determinando el rango, promedio y desviación estándar (Weisbrot, 1985), verificándo la distribución normal de los datos por la prueba de KolmogorovSmirnov,

Se determinó el rango de referencia para cada metabolito implicados en el estudio utilizando la metodología descrita por Bermes et al., (1976) y Weisbrot (1985), obteniendo los intervalos de confianza al 95\%. Además, se realizaron correlaciones de Pearson y Stephens (1964), para determinar el grado de asociación entre las variables peso, condición corporal y etapas de la gestación con los valores de los metabolitos en estudio.

\section{Resultados y discusión}

Los valores promedios totales, el error estándar y los intervalos de confianza de los metabolitos analizados se observan en la Tabla 1. Los valores promedio totales de Glu $(43,76 \mathrm{mg} / \mathrm{dL})$ fueron inferiores a los reportados en razas criollas colombianas $(46.98 \mathrm{mg} / \mathrm{dL})$ (Campos et al., 2004), vacas Jersey $(48,1 \mathrm{ng} / \mathrm{dL})$ a los 8 dias PP (Roos et al., 2008), vacas rubia Gallega al primer mes posparto $(57,38 \mathrm{mg} / \mathrm{dL}$ ) (Quintela et al., 2011) y en general para la especie $(45-75 \mathrm{mg} / \mathrm{dl})(\mathrm{Ka}-$ neko, 1989).

Los valores de Glu fueron diferentes para los tres trimestres de gestación $(\mathrm{P}<0,05)$, Tabla 2 , encontrando los mayores valores en T2 $(47,43 \mathrm{mg} / \mathrm{dl})$, estos niveles pueden ser atribuibles a una mayor demanda por parte del feto. Consecuentemente los menores valores en T1 $(37,04 \mathrm{ng} / \mathrm{dL})$ y T3 $(43,63 \mathrm{mg} / \mathrm{dL})$ podrían estar asociados en el primer caso a causas nutricionales, ya que este periodo coincidió con la época de sequía en donde las deficiencias de energía y proteína fueron marcadas debido a la escasez de nutrientes en el forraje y en el segundo caso, a la alta demanda por parte del feto en donde el crecimiento fetal es mayor. Los valores en T3 son menores a los reportados en vacas 
Brahman (50,81 ng/dL) durante las últimas semanas de gestación (Villa et al., 2011).

Los niveles de Glu presentaron una correlación negativa $(r=-0,388 ; P<0,01)$ con el BUN es decir, que cuando la Glu aumentaba el BUN disminuía. Obeidat et al., 2002 asociaron los elevados niveles de nitrógeno ureico en suero con un bajo consumo energético o con condiciones severas de desnutrición.

Contrariamente, la Glu plasmática presentó una correlación positiva con la AST $(r=0,316 ; \mathrm{P}<0,01)$, es decir que cuando aumentaba la Glu aumentaba la AST. Dado que la síntesis de Glu sanguínea en los rumiantes depende totalmente de la función hepática, cualquier alteración en la actividad de las enzimas indicadoras del daño hepático, tienen mayor valor diagnóstico que la propia glicemia (Álvarez, 2008).

El Col no mostró diferencias $(\mathrm{P}>0,05)$ durante los trimestres de gestación, sin embargo en T3, los niveles fueron más bajos (Tabla 2), coincidiendo con los arreglos metabólicos previos al parto como la movilización de la grasa, crecimiento fetal y el inicio de la lactancia (Bell 1995). Una tendencia similar fue encontrada por Shope y Gowen (1928), quienes a su vez observaron una gran variación individual. Los niveles de Col en función de grupo racial presentaron diferencias

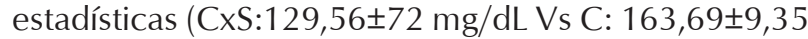
$\mathrm{mg} / \mathrm{dL} \mathrm{P}<0,05)$ lo cual sugiere que el ganado Cebú podrían tener mayores valores de Col en sangre, como ha sido sugerido (Álvarez, 2008).

Barrios et al., 2013 encontraron en la región centro occidental de Venezuela una media de $118 \pm 35 \mathrm{mg} /$ dL para el Col, valor muy inferior al encontrado en este estudio. De igual forma, Giraldo et al., 2008 encontraron bajos niveles de Col en vacas Brahman (108,2mg/ $\mathrm{dL})$ cuatro semana antes del parto con una $\mathrm{CC} \leq \mathrm{a} 8$.

Respecto a las Ptt los valores promedio se encontraron por debajo del rango reportado para la especie (6,74-74,6 ng/dL). Además no se encontró diferencias en los niveles por trimestre de gestación. En tanto que la síntesis de proteínas esta bajo control genético, se esperaría encontrar diferencias entre los dos grupos raciales, sin embargo esta situación no fue observada.

Durante la gestación el total de las Ptt disminuyen debido a una disminución de la Alb y un ligero incremento de las Glob, mas cerca al parto hay un rápido incremento en las a-globulinas y un aumento en la totalidad de las Ptt plasmáticas; en la lactación el total de las proteínas plasmáticas disminuye debido a la disminución de la Alb (Kaneko, 1989). Los niveles de Alb (3,06ng/dL) encontrados en nuestro estudio, se

Tabla 1. Concentraciones medias, error estándar e IC para los metabolitos estudiados en vacas de un sistema de producción de cría en la Altillanura colombiana.

\begin{tabular}{|c|c|c|c|}
\hline Metabolito & $\mathbf{N}$ & $\mathrm{X} \pm \mathrm{EE}$ & $\begin{array}{c}\text { IC } \\
\text { Límite Inferior-superior }\end{array}$ \\
\hline Glucosa (mg/dL) & 57 & $43,76 \pm 1,71$ & $40,30-47,23$ \\
\hline Colesterol total (mg/dL) & 57 & $146,21 \pm 7,54$ & $130,99-161,43$ \\
\hline Proteina Total $(\mathrm{g} / \mathrm{dL})$ & 57 & $6,1 \pm 0,12$ & $5,85-6,34$ \\
\hline Albúmina (g/dL) & 57 & $3,06 \pm 0,74$ & $2,91-3,21$ \\
\hline Globulina (g/dL) & 57 & $3,03 \pm 0,13$ & $2,76-3,31$ \\
\hline Hemoglobina $(\mathrm{g} / \mathrm{dL})$ & 57 & $12,76 \pm 0,23$ & $12,29-13,24$ \\
\hline Hematocrito (\%) & 57 & $40,26 \pm 0,44$ & $39,36-41,16$ \\
\hline BUN (mg/dL) & 57 & $10,62 \pm 0,60$ & $9,4-11,83$ \\
\hline Cobre $(\mathrm{mg} / \mathrm{dL})$ & 57 & $26,59 \pm 1,38$ & $23,81-29,38$ \\
\hline Zinc $(\mathrm{mg} / \mathrm{dL})$ & 56 & $31,63 \pm 2,98$ & $25,62-37,64$ \\
\hline Calcio (mg/dL) & 57 & $10,38 \pm 0,36$ & $9,66-11,11$ \\
\hline Magnesio (mg/dL) & 57 & $2,76 \pm 0,33$ & $2,1-3,43$ \\
\hline Fósforo $(\mathrm{mg} / \mathrm{dL})$ & 44 & $5,71 \pm 0,23$ & 5,23-619, \\
\hline AST (UI) & 57 & $60,32 \pm 1,89$ & $56,50-64,14$ \\
\hline Peso vivo (kg) & 57 & $519,89 \pm 6,5$ & $506,76-533,0$ \\
\hline CC (1-9) & 57 & $8,12 \pm 0,45$ & $8,03-8,21$ \\
\hline
\end{tabular}

$\diamond$ Intervalo de confianza (95\%). 
Tabla 2. Concentraciones medias, desviación estándar y rango para los metabolitos energéticos y nitrogenados durante los trimestres de la gestación en vacas de cría en la Altillanura colombiana.

\begin{tabular}{|c|c|c|c|c|}
\hline $\begin{array}{c}\text { Metabolito } \\
(\mathrm{mg} / \mathrm{dL})\end{array}$ & $\begin{array}{l}\text { Tercio de la } \\
\text { gestación }\end{array}$ & $\mathbf{N}$ & $X \pm D E$ & $\begin{array}{c}\text { IC }^{\diamond} \\
\text { Límite inferior-superior }\end{array}$ \\
\hline Glucosa (mg/dL) & $\begin{array}{l}\text { T1 } \\
\text { T2 } \\
\text { T3 }\end{array}$ & $\begin{array}{l}19 \\
19 \\
19\end{array}$ & $\begin{array}{c}37,04 \pm 2,29^{a} \\
47,43 \pm 2,48^{b} \\
43,63 \pm 2,32^{a b}\end{array}$ & $\begin{array}{l}32,22-41,85 \\
42,20-52,66 \\
38,75-48,51\end{array}$ \\
\hline $\begin{array}{l}\text { Colesterol total } \\
(\mathrm{mg} / \mathrm{dL})\end{array}$ & $\begin{array}{l}\text { T1 } \\
\text { T2 } \\
\text { T3 }\end{array}$ & $\begin{array}{l}19 \\
19 \\
19 \\
\end{array}$ & $\begin{array}{c}147,38 \pm 11,33^{\mathrm{a}} \\
156,68 \pm 9,4^{\mathrm{a}} \\
138,52 \pm 14,17^{\mathrm{a}}\end{array}$ & $\begin{array}{l}123,56-171,20 \\
136,89-176,46 \\
108,74-168,30\end{array}$ \\
\hline $\begin{array}{l}\text { Proteínas } \\
\text { totales }(\mathrm{g} / \mathrm{dL})\end{array}$ & $\begin{array}{l}\mathrm{T} 1 \\
\mathrm{~T} 2 \\
\mathrm{~T} 3 \\
\end{array}$ & $\begin{array}{l}19 \\
19 \\
19 \\
\end{array}$ & $\begin{array}{l}5,73 \pm 0,23^{a} \\
6,16 \pm 0,15^{a} \\
6,28 \pm 0,15^{a}\end{array}$ & $\begin{array}{l}5,25-6,21 \\
5,83-6,50 \\
5,97-6,60 \\
\end{array}$ \\
\hline $\begin{array}{l}\text { Albúmina } \\
(\mathrm{g} / \mathrm{dL})\end{array}$ & $\begin{array}{l}\text { T1 } \\
\text { T2 } \\
\text { T3 } \\
\end{array}$ & $\begin{array}{l}19 \\
19 \\
19 \\
\end{array}$ & $\begin{array}{l}3,17 \pm 0,09^{\underline{a}} \\
2,97 \pm 0,12^{\underline{a}} \\
3,13 \pm 0,10 \mathrm{a} \\
\end{array}$ & $\begin{array}{l}2,97-3,38 \\
2,72-3,23 \\
2,91-3,34 \\
\end{array}$ \\
\hline $\begin{array}{l}\text { Globulina } \\
(\mathrm{g} / \mathrm{dL})\end{array}$ & $\begin{array}{l}\text { T1 } \\
\text { T2 } \\
\text { T3 } \\
\end{array}$ & $\begin{array}{l}19 \\
19 \\
19 \\
\end{array}$ & $\begin{array}{l}2,56 \pm 0,22^{\underline{a}} \\
3,19 \pm 0,18^{\underline{a}} \\
3,16 \pm 0,15^{\underline{a}}\end{array}$ & $\begin{array}{l}2,08-3,04 \\
2,80-3,59 \\
2,83-3,48 \\
\end{array}$ \\
\hline $\begin{array}{l}\text { Hemoglobina } \\
(\mathrm{g} / \mathrm{dL})\end{array}$ & $\begin{array}{l}\text { T1 } \\
\text { T2 } \\
\text { T3 }\end{array}$ & $\begin{array}{l}19 \\
19 \\
19 \\
\end{array}$ & $\begin{array}{l}11,06 \pm 0,20^{\mathrm{a}} \\
13,16 \pm 0,14^{\mathrm{b}} \\
14,17 \pm 0,16^{\mathrm{c}}\end{array}$ & $\begin{array}{l}10,64-11,49 \\
12,86-13,46 \\
13,82-14,52 \\
\end{array}$ \\
\hline $\begin{array}{l}\text { Hematocrito } \\
(\%)\end{array}$ & $\begin{array}{l}\text { T1 } \\
\text { T2 } \\
\text { T3 } \\
\end{array}$ & $\begin{array}{l}19 \\
19 \\
19 \\
\end{array}$ & $\begin{array}{l}38,03 \pm 0,53^{\mathrm{a}} \\
39,62 \pm 0,41^{\mathrm{b}} \\
42,84 \pm 0,51^{\mathrm{c}}\end{array}$ & $\begin{array}{l}36,91-39,15 \\
38,75-40,48 \\
41,76-43,93 \\
\end{array}$ \\
\hline $\begin{array}{l}\text { BUN } \\
(\mathrm{mg} / \mathrm{dL})\end{array}$ & $\begin{array}{l}\text { T1 } \\
\text { T2 } \\
\text { T3 }\end{array}$ & $\begin{array}{l}19 \\
19 \\
19\end{array}$ & $\begin{array}{c}15,36 \pm 0,30^{b} \\
7,41 \pm 0,41^{a} \\
8,44 \pm 0,43^{a}\end{array}$ & $\begin{array}{c}14,71-16,01 \\
6,53-8,28 \\
7,53-9,34\end{array}$ \\
\hline
\end{tabular}

$\diamond$ Intervalo de confianza (95\%).

encontró dentro de los valores de referencia para la especie (3,03-3,55 ng/dL). Al igual que la Alb y las Glo no se encontraron diferencia entre los trimestres de gestación, ni por grupo racial. Generalmente un perfil anormal de proteínas se ha asociado con alguna enfermedad (Kaneko, 1989), situación que no fue observado en este estudio.

A medida que avanzaba la gestación se encontró un aumento en la Hem y el Hto, encontrándo diferencias $(P<0,05)$ entre los tercios de la gestación (Tabla 2). Los valores promedios obtenidos para la Hem y Hto en T3 son mayores a los reportados por Zambrano y Marques (2009) en vacas Gyrolando, atribuidos posiblemente a una mayor demanda de aminoácidos necesarios para la síntesis de proteínas, que hace reducir la síntesis de otras proteínas (Contreras, 2000). De forma contraria se ha visto que la Hem disminuye significativamente durante la mitad de la gestación con los menores valores durante el segundo mes (Calvo et al., 1989), esta disminución ocurre por el ingreso de la
Hem de la madre dentro de la circulación fetal, apoyada por la dilución de la sangre como consecuencia del aumento del volumen del plasma (Singh et al., 1991).

Los valores del Hto de este estudio fueron superiores a lo reportado en vacas Brahman y cruzadas Brahman $x$ Sanmartinero que pastaban sabanas nativas similares a las utilizadas por los animales de este estudio (Vera y Rámirez-Restrepo, 2017). Con respecto a la Hem los valores son mayores a los encontrados en vacas Sahiwal durante los trimestres de gestación (Roy et al., 2010). De todas formas los resultados tanto de la Hem y Hto encontrados en nuestro estudio deben ser analizados con cuidado puesto que se conoce que la bioquímica sanguínea y los componentes celulares se ven influenciados por varios factores entre ellos, la raza, el sexo, la edad, el comportamiento, el manejo, los cambios fisiológicos y el periodo del dia (Taylor-Macallister, 1997). Las Ptt presentaron una correlación positiva con las Glo $(r=0,848 ; \mathrm{P}<0,01)$, mientras se observó una correlación negativa entre la Alb y Glo $(r=-0,383 ; P<0,01)$ 
Se encontró una correlación positiva entre la Hem y Hto $(r=0,781 ; P<0,01)$. De igual forma, estos dos metabolitos presentaron una correlación positiva con la CC y el PV $(r=0,510, r=0,397 ; P<0,01$ respectivamente). De forma contraria, el BUN presentó una correlación negativa con la Hem, Hto, CC, y PV ( $r=-0,711$, $r=-0,419, r=-0,430$ y $r=-0,441 ; P<0,01$ respectivamente) y una correlación positiva con el $\mathrm{Ca}(\mathrm{r}=0,275$; $\mathrm{P}<0,05)$.

El BUN varió para los diferentes trimestres de gestación $(\mathrm{P}<0,05)$, (Tabla 2) presentando las concentraciones mas altas en T1, periodo que coincidió con la época final de la sequía, la cual es crítica para el crecimiento de las pasturas en esta región del país. Las concentraciones más bajas de BUN se observaron en T2 y T3, lo que puede estar relacionado con la baja calidad del pasto (época seca). Sin embargo, niveles altos de BUN pueden estar asociados a la oferta de Proteína cruda (PC) ofrecida durante el pastoreo, al bajo consumo de energía y/o condiciones severas de desnutrición (Obeidat et al., 2002), se puede inferir entonces, que los altos niveles de BUN durante T1 pudieron estar influenciados por la cantidad de PC presente en la pasturas y el consumo de energía (EE), descartando un estado de desnutrición ya que, los animales presentaron una buena condición corporal $(7,93 \pm 0,60)$. Los niveles de BUN durante T1 son similares a los reportados por Tabeleão et al., 2006 en hembras mestizas (Bos taurus x Bos Indicus) en Río Grande/RS, Brasil y superiores a los reportados por Ceballos et al., 2002 en ganado lechero del trópico alto del eje cafetero colombiano e inferiores a las encontradas durante el preparto en vacas Rubia gallega (Quintela et al., 2011). Resultados similares para el BUN encontrados en este estudio han sido reportados por Romero-Treviño 2011 en vacas multíparas Pardo Suizo x Cebú en lactancia con una concentración de 10.6 mg/dL e inferior a la reportada por Barrios et al., 2013. Respecto a los niveles de acuerdo con el grupo racial no se encontró diferencia $(p>0.05)$.

Las concentraciones séricas de hemoglobina, hematocrito y BUN, se observaron variaciones según el tercio de la gestación (Tabla 2).

Los niveles promedios de Cu y Zn encontrados en este estudio, están por debajo de los niveles críticos $(\mathrm{Cu}=$ $<0,57 \mathrm{mg} / \mathrm{ml} ; \mathrm{Zn}=<0.6 \mathrm{mg} / \mathrm{ml}$ ) (Noaman et al., 2012) para todos los trimestres de gestación evaluados sin diferencias entre trimestres ni por grupo racial.

Otros estudios han encontrado bajos niveles de $\mathrm{Cu}$ en vacas preñadas que coinciden con nuestro estudio, lo cual se ha atribuido a que la preñez es dependiente del $\mathrm{Cu}$, dadas las demandas adicionales para atender esta condición (Noaman et al., 2012). Estos resulta- dos coinciden a la vez con los bajos niveles al final de la gestación (Slavick et al., 2006). Es conocido que el Cu es importante para diversas funciones corporales como la formación de hemoglobina, crecimiento, prevención de trastornos patológicos, actividad de las metaloenzimas esenciales en la reproducción normal, entre otras (Underwood y Suttle, 1999), por lo que las deficiencias de este mineral puede afectar estos procesos.

Estudios en EU en un sistema de producción vacaternero bajo condiciones similares a nuestro estudio, encontraron que los forrajes no eran normales en $\mathrm{Zn}$ y marginales en Cu con baja disponibilidad de $\mathrm{Cu}$ debido a las altas concentraciones de Fe y Mo (Corah et al., 1996; Mortimer et al., 1999). Esta situación es concordante bajo las condiciones de la Altillanura colombiana, caracterizadas por los altos niveles de Fe. De otro lado se ha asociado las deficiencias de Cu con los bajos niveles contenidos en el alimento (Ward, 1978)

Respecto al Zn, más de 200 enzimas dependen de este mineral, por lo que participa en la biosintesis de acidos nucleicos, el desarrollo esquelético, la estabilizacion del DNA, RNA y ribosomas, la replicación y diferenciación celular, el metabolismo de carbohidratos, síntesis y actividad de hormonas entre otras (Ahola, 2004). En Ganado alimentado con una dieta baja en $\mathrm{Zn}$, la rata de absorción solo fue de 50\% (Stake et al., 1975). Los bajos niveles de Cu y Zn podría sugerir alguna deficiencia subclinica sin que se reconocieran desórdenes clínicos en los animales analizados, tal como ha sido observado en otro estudio (Fiorentin et al., 2018). Otra consideración importante y que no fue analizada en este estudio es la variación que presentan estos minerales de acuerdo con la época. De tal forma, se han encontrado elevadas concentraciones sericas de Cu durante el invierno frente al verano (Noaman et al., 2012), lo que plantea el interrogante si se debería usar una sal mineralizada con la misma composición química todo el año.

Los niveles de Ca, Mg y $\mathrm{P}$ se encontraron dentro de los niveles normales sugeridos por González y Silva, 2006 y Álvarez 2008 y los encontrados por Barrios et al., 2013. Los valores de Cu $(26,59 \pm 1,38 \mathrm{mg} / \mathrm{dL})$ y $\mathrm{Zn}$ $(31,63 \pm 2,98 \mathrm{mg} / \mathrm{dL})$ no presentaron diferencias para el tipo racial (tabla 5), aunque se consideran como valores críticos para los bovinos.

En este caso, la aparente discrepancia entre los valores séricos bajos, niveles al límite y superior de Zn e inferior de $\mathrm{Cu}$ en las praderas, posiblemente se debe a las altas concentraciones de Fe en el suelo y el forraje que impiden una mejor disponibilidad de $\mathrm{P}$ para las 
Tabla 3. Concentraciones medias, desviación estándary rango para los metabolitos energéticos y nitrogenados encontrados durante la gestación en vacas cruzadas Cebú x Simmental (CxS) y Cebú (C), de un sistema de producción de cría en la Altillanura colombiana.

\begin{tabular}{|c|c|c|c|c|}
\hline $\begin{array}{c}\text { Metabolito } \\
(\mathrm{mg} / \mathrm{dL})\end{array}$ & $\begin{array}{c}\text { Predominio } \\
\text { racial }\end{array}$ & $\mathbf{N}$ & $\mathrm{X} \pm \mathrm{DE}$ & $\begin{array}{c}\qquad C^{\diamond} \\
\text { Límite inferior-superior }\end{array}$ \\
\hline Glucosa & $\begin{array}{c}\mathrm{CxS} \\
\mathrm{C}\end{array}$ & $\begin{array}{l}27 \\
30 \\
\end{array}$ & $\begin{array}{l}41,47 \pm 1,84^{\mathrm{a}} \\
43,81 \pm 2,23^{\mathrm{a}}\end{array}$ & $\begin{array}{l}37,29-46,43 \\
40,11-50,89 \\
\end{array}$ \\
\hline Colesterol Total & $\begin{array}{c}\mathrm{CxS} \\
\mathrm{C}\end{array}$ & $\begin{array}{l}27 \\
30 \\
\end{array}$ & $\begin{array}{l}129,56 \pm 8,72^{\mathrm{a}} \\
163,69 \pm 9,35^{\mathrm{b}}\end{array}$ & $\begin{array}{c}112,48-151,89 \\
136,0-182,04 \\
\end{array}$ \\
\hline Proteína total $(\mathrm{g} / \mathrm{dL})$ & $\begin{array}{c}\mathrm{CxS} \\
\mathrm{C}\end{array}$ & $\begin{array}{l}27 \\
30 \\
\end{array}$ & $\begin{array}{c}6,0 \pm 0,16^{\mathrm{a}} \\
6,11 \pm 0,14^{\mathrm{a}} \\
\end{array}$ & $\begin{array}{l}5,53-6,38 \\
5,92-6,53 \\
\end{array}$ \\
\hline Albúmina (g/dL) & $\begin{array}{c}\mathrm{CxS} \\
\mathrm{C}\end{array}$ & $\begin{array}{l}27 \\
30 \\
\end{array}$ & $\begin{array}{l}3,06 \pm 0,09^{a} \\
3,12 \pm 0,79^{a} \\
\end{array}$ & $\begin{array}{l}2,75-3,25 \\
2,93-3,31 \\
\end{array}$ \\
\hline Globulina (g/dL) & $\begin{array}{c}\mathrm{CxS} \\
\mathrm{C}\end{array}$ & $\begin{array}{l}27 \\
30 \\
\end{array}$ & $\begin{array}{c}2,94 \pm 0,17^{\mathrm{a}} \\
3,0 \pm 0,15^{\mathrm{a}} \\
\end{array}$ & $\begin{array}{l}2,49-3,43 \\
2,77-3,44 \\
\end{array}$ \\
\hline Hemoglobina $(\mathrm{g} / \mathrm{dL})$ & $\begin{array}{c}\mathrm{CxS} \\
\mathrm{C}\end{array}$ & $\begin{array}{l}27 \\
30 \\
\end{array}$ & $\begin{array}{l}12,76 \pm 0,26^{\mathrm{a}} \\
12,83 \pm 0,30^{\mathrm{a}}\end{array}$ & $\begin{array}{l}11,91-13,18 \\
13,69-12,99 \\
\end{array}$ \\
\hline Hematocrito (\%) & $\begin{array}{c}\mathrm{CxS} \\
\mathrm{C}\end{array}$ & $\begin{array}{l}27 \\
30 \\
\end{array}$ & $\begin{array}{l}40,04 \pm 0,43^{a} \\
40,28 \pm 0,62^{a}\end{array}$ & $\begin{array}{l}38,83-40,84 \\
39,13-42,18 \\
\end{array}$ \\
\hline BUN (mg/dL) & $\begin{array}{c}\mathrm{CxS} \\
\mathrm{C}\end{array}$ & $\begin{array}{l}27 \\
30\end{array}$ & $\begin{array}{l}10,49 \pm 0,76^{\mathrm{a}} \\
10,32 \pm 0,72^{\mathrm{a}}\end{array}$ & $\begin{array}{c}14,71-16,01 \\
8,39-11,70 \\
\end{array}$ \\
\hline
\end{tabular}

$\diamond \quad$ Intervalo de confianza (95\%).

plantas, de igual forma, se presenta una baja absorción de Cu y Zn en el ganado (Fordyce et al., 1996), resultados que se podrían asimilar a los encontrados en este estudio.

Por otro lado, se conoce que el Fe compite por los sitios de absorción a nivel intestinal con el Cu. La presencia en este estudio de animales con niveles de $\mathrm{Cu}$ y $\mathrm{Zn}$ por debajo de sus requerimientos hace pensar que estarían predispuestos a desarrollar una carencia secundaria de cobre.

La concentración del Zn sérico presentó una correlación negativa con la AST $(r=-0,299 ; \mathrm{P}<0,05)$, lo que indica que aumentos de la AST se corresponden con disminuciones del Zn. De igual forma el Ca con el Zn $(r=-0,29 ; P<0,05)$ y una correlación positiva de este último con el BUN ( $r=0,275 ; \mathrm{P}<0,05)$.

La concentración total de Ca $(10,3 \pm 0,3 \mathrm{ng} / \mathrm{dL})$ fue superior a los limites reportados para la especie $(8,0-10,5$ $\mathrm{mg} / \mathrm{dL})$ con los mayores valores en $\mathrm{T} 1(11,98 \mathrm{mg} / \mathrm{dL})$ y T3 $(11,38 \mathrm{mg} / \mathrm{dL})$ los cuales fueron diferentes a T2 $(9,09 \mathrm{mg} / \mathrm{dL})(\mathrm{P}<0,05)$ muy por debajo del límite inferior (Tabla 4), indicando tal vez una mayor demanda y consumo en este trimestre. Sin embargo, los niveles séricos de BUN y Ca durante T2 pueden estar indican- do una carencia de proteína bruta y de Ca o una mayor demanda por parte del feto durante este trimestre.

Durante T1 y T3 los valores séricos del Ca siempre se mantuvieron por encima de los limites normales (8,5$9,0 \mathrm{mg} / \mathrm{dl}$ ) (Kaneko et al., 1997). Con nivel deficiente de Ca en el suelo, un porcentaje de Ca bajo durante la época de lluvias y un porcentaje superior durante la sequía de las pasturas, los valores séricos encontrados en este estudio se deben fundamentalmente al eficiente mecanismo homeostático que mantuvo el nivel del Ca sérico dentro de límites fisiológico (Said et al., 1964.) y a la suplementación mineral en fósforo que recibieron los animales (sal mineralizada al 8\%).

Se ha identificado que el exceso de Ca disminuye la absorción de Zn (Graham 1991), por lo que es posible que esta situación haya ocurrido en este estudio, sin que se observe que los niveles de Ca durante la gestación variarán significativamente (Tabla. 4) pudiendo afectar la absorción del Zn. Durante T1 (época seca), los valores séricos de $\mathrm{Cu}, \mathrm{Ca}$ y $\mathrm{P}$ resultaron mayores (Tabla 4), y superiores a los reportados por Depablos et al., 2009. Los valores de Mg no presentaron diferencias por trimestre de gestación, ni por grupo racial y se encontraron dentro de los niveles normales para vacas de carne (1,2-3,5 ng/dL) (Church et al., 1978). Similar comportamiento presentaron los niveles de fosforo. 
Tabla 4. Concentraciones medias, desviación estándar y rango para los metabolitos minerales encontrados durante los trimestres de la gestación en vacas de cría en la Altillanura Colombiana.

\begin{tabular}{|c|c|c|c|c|}
\hline $\begin{array}{c}\text { Metabolito } \\
(\mathrm{mg} / \mathrm{dL})\end{array}$ & $\begin{array}{c}\text { Tercio de la } \\
\text { gestación }\end{array}$ & $\mathbf{N}$ & $X \pm D E$ & $\mathrm{IC}^{\diamond}$ \\
\hline Cobre & $\begin{array}{l}\text { T1 } \\
\text { T2 } \\
\text { T3 }\end{array}$ & $\begin{array}{l}19 \\
19 \\
19\end{array}$ & $\begin{array}{l}29,03 \pm 2,83^{\mathrm{a}} \\
23,02 \pm 1,50^{\mathrm{a}} \\
28,76 \pm 0,85^{\mathrm{a}}\end{array}$ & $\begin{array}{l}23,06-34,99 \\
19,85-26,19 \\
26,97-30,55\end{array}$ \\
\hline Zinc & $\begin{array}{l}\mathrm{T} 1 \\
\mathrm{~T} 2 \\
\mathrm{~T} 3\end{array}$ & $\begin{array}{l}19 \\
19 \\
18\end{array}$ & $\begin{array}{l}30,25 \pm 2,62^{a} \\
36,92 \pm 6,18^{a} \\
29,46 \pm 2,39^{a}\end{array}$ & $\begin{array}{l}24,72-35,77 \\
23,94-49,91 \\
24,41-34,51\end{array}$ \\
\hline Calcio & $\begin{array}{l}\text { T1 } \\
\text { T2 } \\
\text { T3 }\end{array}$ & $\begin{array}{l}19 \\
19 \\
19\end{array}$ & $\begin{array}{c}11,98 \pm 0,77^{b} \\
9,09 \pm 0,52^{a} \\
11,38 \pm 1,77^{b}\end{array}$ & $\begin{array}{c}10,37-13,60 \\
7,99-10,19 \\
10,53-12,23\end{array}$ \\
\hline Magnesio & $\begin{array}{l}\text { T1 } \\
\text { T2 } \\
\text { T3 }\end{array}$ & $\begin{array}{l}19 \\
19 \\
19\end{array}$ & $\begin{array}{l}2,50 \pm 0,71^{\mathrm{a}} \\
2,76 \pm 0,25^{\mathrm{a}} \\
2,78 \pm 0,24^{\mathrm{a}}\end{array}$ & $\begin{array}{c}1,0-4,0 \\
2,23-3,29 \\
2,27-3,30\end{array}$ \\
\hline Fósforo & $\begin{array}{l}\text { T1 } \\
\text { T2 } \\
\text { T3 }\end{array}$ & $\begin{array}{l}16 \\
16 \\
12\end{array}$ & $\begin{array}{l}5,96 \pm 0,34^{a} \\
5,61 \pm 0,37^{a} \\
5,50 \pm 0,56^{a}\end{array}$ & $\begin{array}{l}5,23-6,70 \\
4,80-6,42 \\
4,25-6,75\end{array}$ \\
\hline
\end{tabular}

$\checkmark \quad$ Intervalo de confianza (95\%).

La concentración promedio de $\mathrm{P}$ sérico durante la gestación y durante los trimestres de la gestación se encontraron dentro de los rangos sugeridos para ganado bovino (Kaneko et al., 1997). Con deficiencias de fósforo en suelo y en las pasturas, los valores normales séri- cos de fósforo se podrían explicar por suplementación mineral en fósforo recibida por los animales durante el estudio (sal mineralizada al $8 \%$ ). En general se puede decir que los minerales deben ser analizados en su conjunto, debido a sus múltiples interacciones, tal como lo

Tabla 5. Concentraciones medias, desviación estándar y rango para los metabolitos minerales encontrados durante la gestación en vacas cruzadas Cebú x Simmental (CxS) y Cebú (C), de un sistema de producción de cría en la Altillanura colombiana.

\begin{tabular}{|c|c|c|c|c|}
\hline $\begin{array}{c}\text { Metabolito } \\
(\mathrm{mg} / \mathrm{dL})\end{array}$ & $\begin{array}{c}\text { Predominio } \\
\text { racial }\end{array}$ & $\mathbf{N}$ & $X \pm D E$ & $\mathrm{IC}^{\diamond}$ \\
\hline Cobre & $\begin{array}{c}\mathrm{CxS} \\
\mathrm{C}\end{array}$ & $\begin{array}{l}27 \\
30 \\
\end{array}$ & $\begin{array}{l}28,64 \pm 2,20^{\mathrm{a}} \\
25,40 \pm 0,87^{\mathrm{a}}\end{array}$ & $\begin{array}{l}23,01-33,96 \\
22,66-27,07 \\
\end{array}$ \\
\hline Zinc & $\begin{array}{c}\mathrm{CxS} \\
\mathrm{C}\end{array}$ & $\begin{array}{l}26 \\
30\end{array}$ & $\begin{array}{l}35,35 \pm 4,76^{a} \\
29,58 \pm 1,71^{a}\end{array}$ & $\begin{array}{l}23,40-47,95 \\
24,24-31,63\end{array}$ \\
\hline Calcio & $\begin{array}{c}\mathrm{CxS} \\
\mathrm{C}\end{array}$ & $\begin{array}{l}27 \\
30\end{array}$ & $\begin{array}{l}10,76 \pm 0,53^{a} \\
10,87 \pm 0,52^{a}\end{array}$ & $\begin{array}{l}9,14-11,67 \\
9,47-11,25\end{array}$ \\
\hline Magnesio & $\begin{array}{c}\mathrm{CxS} \\
\mathrm{C}\end{array}$ & $\begin{array}{l}27 \\
30\end{array}$ & $\begin{array}{l}2,61 \pm 0,18^{a} \\
2,74 \pm 0,47^{a}\end{array}$ & $\begin{array}{l}2,08-2,99 \\
1,72-4,22\end{array}$ \\
\hline Magnesio & $\begin{array}{c}\mathrm{CxS} \\
\mathrm{C}\end{array}$ & $\begin{array}{l}21 \\
23\end{array}$ & $\begin{array}{l}5,69 \pm 0,33^{a} \\
5,73 \pm 0,34^{a}\end{array}$ & $\begin{array}{c}5,0-6,38 \\
5,01-6,44\end{array}$ \\
\hline
\end{tabular}

$\checkmark \quad$ Intervalo de confianza (95\%). 
demostró un estudio en ratas preñadas en donde la deficiencia de Fe tuvo efectos opuestos sobre los niveles de Cu y Zn en el hígado fetal, lo cual puede afectar el metabolismo de estos nutrientes con graves consecuencias para el desarrollo fetal y el neonato (Cottin et al., 2019).

No se encontraron diferencias significativas en la concentración sanguínea de la AST durante los trimestres de la gestación, ni por grupo racial (Tabla 6 y 7). La AST es un buen indicador del funcionamiento hepático, aumentos de la AST sérica se encuentran asociados con daño hepático, como en el caso de las hipoglicemias (González y Silva, 2006).

Se presentó un aumento de la AST a medida que avanzaba la gestación (tabla 6). La concentración sérica promedio de AST encontrada en este estudio fue superior a la reportada por Roos et al., 2008 en vacas Jersey al sur de Rio Grande do Sul e inferior a las encontradas por Quintela et al., 2011 en vacas de la raza Rubia Gallega y por Coppo et al., en 2000 en bovinos cruzados Cebú x Europeo (30UI/L). Contrariamente la disminución de la AST a través de la gestación, se ha asociado con cambios hormonales y uterinos (Roy et al., 2010).

Barini 2007, en un estudio con ganado Curraleiro, encontró que los niveles séricos de AST eran directamente proporcionales a la edad, lo que evidencia que los PM se ven influenciados por diferentes factores entre ellos la edad.

Se observaron variaciones en el peso y la CC durante los trimestres de la gestación (Tabla 6), pero no por predominio racial (Tabla 7). Los mayores pesos se observaron en las vacas con predominio racial $\mathrm{CxS}$
$(533,52 \pm 9,90 \mathrm{~kg})$ frente a las Cebú $(520,5 \pm 7,43 \mathrm{~kg})$, contrario a lo encontrado para la CC, en donde las vacas con predominio racial Cebú presentaron la mayor CC $(8,20 \pm 0,43)$ que las CxS $(8,15 \pm 0,72)$. En razón de que tanto el PV como la CC aumentaron a través del tiempo, no es fácil obtener mayor información de la relación de estas variables con los PM, por lo tanto se plantea en futuros estudios conocer esta relación en la etapa de transición y posteriormente en el posparto ya que en estos periodos, la variación de la CC y el PV son mayores, con notaria evidencia de un balance energético negativo, tal como ha sido realizado en otros estudios (Soca et al., 2013).

El PV estuvo altamente relacionado con la CC $(r=0,795 ; P<0,01)$. Datos similares fueron reportados por Villa et al., 2011 durante el periparto en vacas Brahman $(r=0,56 ; P<0,05)$. La promedios de la CC permite afirmar que las vacas mantuvieron sus reservas de grasa para satisfacer los requerimientos energéticos durante la gestación ya que vacas con promedio de CC superior a 6 son animales con suficientes reservas corporales que les permite una mayor resistencia a condiciones de restricción energética.

Al analizar el comportamiento PV y CC frente al resto de metabolitos, se observó una correlación negativa entre la $\mathrm{CC}$ con BUN $(\mathrm{r}=-0,430 ; \mathrm{P}<0,01)$ y positiva con la Hb y el Hto $(r=0,510 ; P<0,01 ; \quad r=0,447 ; P<0,01$ respectivamente). De igual forma, el $\mathrm{PV}$ se correlacionó negativamente con el BUN $(r=-0,441 ; P<0,01)$ y positivamente con la $\mathrm{Hb} \quad(\mathrm{r}=0,397 ; \mathrm{P}<0,01)$ y el Hto $(r=0,258 ; P<0,05)$.

Tabla 6. Concentraciones medias, desviación estándar y rango para los metabolitos AST, peso vivo y CC durante los trimestres de la gestación en vacas de cría en la Altillanura Colombiana.

\begin{tabular}{|c|c|c|c|c|}
\hline Metabolito & $\begin{array}{c}\text { Trimestre de la } \\
\text { gestación }\end{array}$ & $\mathbf{N}$ & $\mathbf{X} \pm \mathbf{D E}$ & IC $^{\boldsymbol{}}$ \\
\hline \multirow{3}{*}{ AST (UI/L) } & T1 & 19 & $55,74 \pm 2,06^{\mathrm{a}}$ & $51,41-60,07$ \\
& T2 & 19 & $60,47 \pm 3,72^{\mathrm{a}}$ & $52,64-68,31$ \\
& T3 & 19 & $63,42 \pm 1,64^{\mathrm{a}}$ & $59,97-66,87$ \\
\hline \multirow{3}{*}{ Peso (kg) } & T1 & 19 & $487,63 \pm 7,32^{\mathrm{a}}$ & $472,23-503$ \\
& T2 & 19 & $538,95 \pm 9,32^{\mathrm{b}}$ & $518,71-559,1$ \\
& T3 & 18 & $553,42 \pm 8,34^{\mathrm{b}}$ & $535,89-570,9$ \\
\hline \multirow{3}{*}{ CC (1-9) } & T1 & 19 & $7,93 \pm 0,60^{\mathrm{a}}$ & $472,23-503$ \\
& T2 & 19 & $8,23 \pm 0,54^{\mathrm{b}}$ & $518,71-559,1$ \\
& T3 & $8,37 \pm 0,58^{\mathrm{b}}$ & $535,89-570,9$ \\
\hline
\end{tabular}

$\checkmark \quad$ Intervalo de confianza (95\%). 
Tabla 7. Concentraciones medias, desviación estándar y rango para AST, peso vivo y condición corporal encontrados durante la gestación en vacas cruzadas Cebú x Simmental (CXS) y Cebú (C), de un sistema de producción de cría en la Altillanura colombiana.

\begin{tabular}{|l|c|c|c|c|}
\hline $\begin{array}{c}\text { Variables } \\
(\mathbf{U I} / \mathbf{L})\end{array}$ & $\begin{array}{c}\text { Predominio } \\
\text { racial }\end{array}$ & $\mathbf{N}$ & $\mathbf{X} \pm \mathbf{D E}$ & $\begin{array}{c}\text { IC }^{\diamond} \\
\text { Límite inferior-superior }\end{array}$ \\
\hline \multirow{2}{*}{ AST } & CXS & 27 & $60,85 \pm 2,67^{\mathrm{a}}$ & $53,88-68,02$ \\
& $\mathrm{C}$ & 30 & $59,0 \pm 1,73^{\mathrm{a}}$ & $55,66-63,82$ \\
\hline \multirow{2}{*}{ Peso (kg) } & CXS & 27 & $533,52 \pm 9,9^{\mathrm{a}}$ & $497,9-540,6$ \\
& $\mathrm{C}$ & 30 & $520,5 \pm 7,43^{\mathrm{a}}$ & $502,9-537,9$ \\
\hline \multirow{2}{*}{ Condición corporal (1-9) } & $\mathrm{CXS}$ & 27 & $8,15 \pm 0,72^{\mathrm{a}}$ & $7,90-8,20$ \\
& $\mathrm{C}$ & 30 & $8,20 \pm 0,43^{\mathrm{a}}$ & $8,09-8,30$ \\
\hline
\end{tabular}

Intervalo de confianza (95\%).

Se concluye finalmente que la mayor parte de los metabolitos analizados se encontraban dentro de los límites normales, excepto el $\mathrm{Cu}$ y $\mathrm{Zn}$, sin que llegaran a observar desórdenes clínicos asociadas a esta deficiencia. Se reconoce el uso de los PM para identificar los problemas asociados a la alimentación y nutrición y se requieren nuevos estudios en una población más amplia, lo cual permita hacer más eficiente el sistema de cría propio de la Altillanura colombiana.

\section{Referencias}

Ahola JK. 2004. Copper, Zinc, and Manganese In Beef Cattle Production: Effects of supplementation and source on reproduction, mineral status, feedlot performance, immunity, and carcass characteristics. Tesis $\mathrm{PhD}$. Colorado State University

Álvarez JL. 2008. Bioquímica nutricional y metabólica del bovino en el tropico. Ed. Universidad de Antioquia, Medellín. p.188

Barth K, Aulrich K, Haufe HC, Muller U, Schaub D, Schulz F. Metabolic status in early lactation dairy cows of two breeds kept under condition of organics farming- a case study. Landbauforschung. VTI agriculture and forestry Research. 2011;4: 61:307-316

Barrios M, Sandoval E, Sánchez D, Borges J, Bastardo Y, Márquez O, Dávila L. 2013. Valores de referencia de diferentes parámetros bioquímicos en vacunos mestizos de doble propósito del valle de aroa, estado Yaracuy. Mundo Pecuario. 2013;9(1):25-30

Bell AW. Regulation of organic nutrient metabolism during transition from late pregnancy to early lactation. J Anim Sci. 1995;73:2804-2819

Bermes-Junior EW, Eeviti V, Forman DT. 1976. Statistics, normal values, and quality control. In: TIETY, N.W. (Ed.). Fundamentals of clinical chemistry. Philadelphia: W.B. Saunders Co.

Butler WR, Smith RD. Interrelationships between energy balance and postpartum reproductive function in dairy cows. J Dairy Sci. 1989;72:767-783

Calvo JJ, Allue JR, Eesudeso A, Garcia LJ. Plasma ferritin of sows during pregnancy and lactation. Cornell Vet. 1989;79:273-282
Campos R, Carreño ES, González FD. Perfil metabólico de vacas nativas colombianas. Orinoquia. 2004;8:32-41

Caton JS, Hess BW. 2010. Maternal plane of nutrition: Impacts on fetal outcomes and postnatal offspring responses. Invited Review. Pages 104-122 in Proc. 4th Grazing Livestock Nutrition Conference.

Ceballos A, Gómez PM, Vélez ML, Villa NA, López LF. Variación de los indicadores bioquímicos del balance de energía según el estado productivo en bovinos lecheros de Manizales. Rev Col de Cienc Pec. 2002;15(1):13-25

Chilliard Y, Bocquier F, Doreau M. Digestive and metabolic adaptations of ruminants to undernutrition and consequences on reproduction. Reprod Nutr Dev. 1998;38:131-152

Church TL, Brunner RR, Janzen ED. A partial metabolic profile in a beef cow herd in which clinical hypocalcemia occurred. Can Vet J. 1978;19(4):110-112

Contreras PA. 2000. Indicadores do metabolismo protéico utilizado nos perfis metabólicos de rebanhos. In: González, F. H. D., J. O. Barcellos, H. Ospina, L. A. O. Ribeiro. (Ed.) Perfil metabólico em ruminantes: seu uso em nutrição e doenças nutricionais. Porto Alegre, Brasil. Gráfica da UFRGS. Pp. 23-30

Conpes. 2014. Política para el desarrollo integral de la Orinoquia: Altillanura - Fase 1. Documento 3797. Consejo Nacional de Política Económica y Social (CONPES), Departamento Nacional de Planeación (DNP), República de Colombia, Bogotá, Colombia.

Coppo JA, Coppo NB, Slanac AL, Revidatti MA, Capellari A. 2000. Influencia del desarrollo, sexo y tipo de destete sobre algumas actividades enzimáticas em plasma de terneros cruza cebú. In: Comunicaciones Científicas y Tecnológicas. Universidad Nacional del Nordeste Argentina.

Corah LR, Dargatz DA, Peters CW. NAHMS Forage Survey: Trace mineral analysis of 352 forage samples collected in 18 states. J Anim Sci. 1996; 74(Suppl. 1):202. (Abstr.)

Cottin SC, Roussel G, Gambling L, Hayes HE, Currie VJ, McArdle HJ. The effect of maternal iron deficiency on zinc and copper levels and on genes of zinc and copper metabolism during pregnancy in the rat. Br J Nutr. 2019;121(2):121-129 
Cutaia L, Brogliatti G, Chesta P, Moreno D, Bó G. 2003. Efecto del momento de la IATF sobre los porcentajes de preñez en vacas para carne sincronizadas con Dispositivos con Progesterona y Benzoato de Estradiol. V ${ }^{\circ}$ Simposio Internacional de Reproducción Animal. Huerta Grande, Córdoba. 27 al 29 de junio. Abstr. 385.

Depablos L, Godoy S, Chicco, CF, Orodoñez J. Nutrición mineral en sistemas ganaderos de las sabanas centrales de Venezuela. Zootecnia Trop. 2009;27(1): 27-38

Edmonson AJ, Lean IJ, Weaver LD, Farver T, Webster G. A body condition scoring chart for Holstein dairy cows. J Dairy Sci. $1989 ; 72: 68-78$

Fiorentin EL, Zanobello S, Gato A, Piovezan AL, Alves MV, Rocha RX, Gonzalez F. Ocurrence of subclinic metabolic disorders in dairy cows from western Santa catarina state, Brasil. Pesq Vet Bras. 2018;38(4):629-634

Fordyce F, Masara D, Appleton J. Stream sediment, soil and forage chemistry as indicators of cattle mineral status in northeast Zimbabwe. Geol Soc. Special Pub. 1996;113:23-37

Freetly HC, Ferrell CL, Jenkins TG. Timing of realimentation of mature cows that were feed-restricted during pregnancy influences calf birth weights and growth rates. J Anim Sci. 2000;78:2790-2796

Giraldo LF, Loaiza AM, Botero SA, Uribe-Velásquez LF. Parámetros metabólicos séricos y condición corporal durante el pre y posparto en vacas Brahman: Serum Metabolic Parameters and Body Condition During Pre and Postpartum in Brahman Cows. Rev Cient. 2009;19(4):350-355

Gómez JM, Laredo MA. Fluctuaciones minerales en pastos tropicales. II. Andropogon gayanus, en los Llanos orientales. Revista ICA. 1983;18:95-104

González FHD, Silva SC. 2006. Introduçao à bioquímica clínica veterinária. Segunda edição. Universidade Federal do Rio Grande do Sul. UFRGS Editora. p. 358

Graham TW. Trace element deficiencies in cattle. Vet Clin North Am: Food Anim Pract. 1991;7:153-215

Houghton PL, Lemanager RP, Horstman LA, Hendrix KS, Moss GE. Effects of body composition, pre- and postpartum energy level and early weaning on reproductive per-formance of beef cows and preweaning calf gain. J Anim Sci. 1990;68:1438-1446

Kaneko JJ. 1989. Clinical biochemistry of domestic animals. San Diego: Academic Press, p. 932

Kaneko JJ, Harvey JW, Bruss ML. 1997. Clinical biochemistry of domestic animals. San Diego: Academic Press p. 932

Kindahl H, Kornmatitsuk B, Königsson K, Gustafsson H. Endocrine changes in late bovine pregnancy with special emphasis on fetal well-being. Dom Anim Endocrinol. 2002; 23(1-2):321-328

Laredo MA, Gómez J. Fluctuaciones minerales en pastos tropicales. I. Brachiaria en los Llanos Orientales. Revista ICA. 1980;15:71-78

Noaman V, Rasti M, Ranjbari AR, Shirvani E. Copper, zinc, and iron concentrations in blood serum and diet of dairy cattle on semi-industrial farms in central Iran. Trop Anim Health Prod. 2012;44:407-411
Mejía GM. 1984. Orinoquia Colombiana: Sabanas de la Altillanura Clima y Uso de la tierra. Fondo Colombiano de Investigaciones Científicas y Proyectos Especiales Francisco José de Caldas Colciencias - Corporación Araracuara. Universidad Nacional de Colombia, Palmira

Mortiner RG, Dargatz DA, Corah LR. 1999. Forage analysis from cow/calf herds in 23 states. USDA:APHIS:VS, Centers for Epidemiology and Animal Health. Fort Collins, CO. \#N303.499. April 1999

Morrison DG, Spitzer JC, Perkins JL. Influence of prepartum body condition score change on reproduction in multiparous beef cows calving in moderate body condition. J Anim Sci. 1999;77:1048-1054

Obeidat BS, Thomas MG, Hallford DM, Keisler DH, Petersen MK, Bryant WD, Garcia MD, Narro L, López R. Metabolic characteristic of multiparous Angus and Brahman cows grazing in the Chihuahuan Desert J Anim Sci. 2002;80(9):2223-2333

Payne JM, Dew SM, Manston R. 1970. The use of a metabolic profile test in dairy herds. Vet Rec.1970;87:150-158

Pearson ES, Stephens MA. 1964.The ratio of range to standard deviation in the same normal sample. Biometrika. 1964;51:484-487

Rae DO, Kunkle WE, Chenoweth PJ, Sand RS, Tran T. 1996. Body condition: influences on beef cattle reproductive performance. AMMVEB (Ed.), XX Congreso Nacional de Buiatría, Acapulco, Gro., México, Pp. 339-343

Rivas L. 2004. Convenio de Cooperación Técnica y Científica MADR - CIAT: Resultados, Adopción e Impacto 1999 - 2003. Documento de Trabajo No 194, Centro Internacional de Agricultura Tropical. Proyecto de Evaluación de Impacto, Cali, Colombia, Julio

Roy S, Roy M, Mishra S. Haematological and biochemical profile during gestation period in Sahiwal cows. Vet World. 2010;3:1:2628

Singh R, Singha SPS, Singh R, Setia MS. Distribution of trace elements in blood, plasma and erythrocytes during different stages of gestation in buffalo (Bubalus Bubalis). Buffalo J. 1991;1:77-85

Smith GL, Friggens NC, Ashworth CJ, Chagunda MGGAssociation between body energy content in the dry period and post-calving production disease status in dairy cattle. Animal. 2017;11:9:1590-1598

Quintela A, Becerra JJ, Rey C, Díaz C, Cainzos J, Rivas F, Huanca W, Prieta A, Herradón PG. 2011. Perfiles metabólicos en preparto, parto y postparto en vacas de raza rubia gallega: estudio preliminar. Recursos Rurais. 2011;7:5-14

Romero-Treviño E M, Gutiérrez-Ornelas E, Bernal-Barragán H, Martínez-González J C, González-Muñoz M A y López-Pérez. Calidad nutricional de pastos, condición corporal y niveles de metabolitos sanguíneos de vacas de doble propósito a través del año en el norte de Veracruz. Liv Res Rural Develop. $2011 ; 23: 215$

Roos TB, Vendramin L, Schwengler E, Goulart MA, Quevedo PS, Silva VM, Verde PML, Del Pino FAB, Timm CD, Gil-Turnes C, Correa MN. Avaliação de parâmetros do perfil metabólico e do leite em diferentes categorias de vacas leiteiras da raça 
Jersey em rebanhos do Sul do Rio Grande do Sul. Vet Foco. 2008;5:121-130

Said RAH, Zaky K, El Azab EA, Shaker M. Effect of age, pregnancy and certain pathological conditions on serum levels of calcium, phosphorus, total protein and protein bound polysaccharides in Fresian cattle. Zentralblatt für Veterinarmendinzin, Ser.A. $1964 ; 11: 685$

Shope RE, Gowen JW. Cholesterol And Cholesterol Ester In The Blood Serum Of Cattle Late In Pregnancy And During The Early Lactation Period. J Exp Med. Jun 1928;30;48(1):25-30

Slavik P, Illek J, Zeleny T. Zinc and copper status of beef cattle in the Sumava region, Czech Republic, Acta Veterinaria Brno. 2006;75:485-488

Soca P, Carriquiry M, Claramun M, Gestido V, Meikle A. Metabolic and endocrine profiles of primiparous beef cows grazing native grassland. 1. Relationships between body condition score at calving and metabolic profiles during the transition period. Animal Production Science. http://dx.doi.org/10.1071/AN13250

Tabeleão VC, Goulart MA, Schwegler E, Weiser MA, Moura SV, Silva VM, Pereira VS, Pino FAB, Corrêa MN. Avaliação ruminal e metabólica de bovinos machos e fêmeas, mantidos em sistema de semi-confinamento. Arch Zootec. 2008;57(218):147-154

Taylor-Macallister C. Haematology and serum biochemistry evaluation in normal postpartum mares. Equine Vet J. 1997;29(3):234-235

Underwood EJ, Suttle NF. 1999. The Mineral Nutrition of Livestock (3rd ed.). CABI Publishing, Wallingford, UK.
Vera RR, Tergas LE. 1986. Develoment of improved grazing system in the savannas of Tropical America. Proc. Second International Rangeland Congress, Adelaide, Australia. P.p. 107-111

Vera RR, Ramírez-Restrepo CA. Complementary use of neotropical savanna and grass-legume pastures for early weaning and effects on growth and metabolic status of weaners and intercalving intervals of dams. Tropical Grasslands-Forrajes Tropicales. 2017;5(2):50-65

Villa NA, Osorio JM, Escobar D, Ceballos A. Indicadores bioquímicos del balance energético en el periparto de vacas Brahman en pastoreo en el trópico colombiano. Rev Cient. 2011;21(4):353-359

Ward G. Molybdenum toxicity and hypocuprosis in ruminants: A review. J Anim Sci. 1978;46:1078-1085

Weisbrot IM. 1985. Statistics for the clinical laboratory. Philadelphia: J.B. Lippincott Company, p. 198

Zambrano JW, Marques jr AP. Perfil metabólico de vacas mestiças leiteiras do pré-parto ao quinto mês da lactação. Zootecnia Trop. 2009;27(4):475-488

Zhang WC, Nakao T, Kida K, Moriyoshi M, Nakada K. Effect of nutrition during pregnancy on calf birth weights and viability and fetal membrane expulsion in dairy cattle. J Reprod Fertil. 2002;48:415-422

\footnotetext{
Gerardo López:https://orcid.org/0000-0002-1283-9490 Agustín Góngora:https://orcid.org/0000-0002-2915-683X Adriana Rojas: https://orcid.org/0001-8678-1028 Félix Díaz:http://orcid.org/0000-0001-9438-6888
} 